\title{
Hemoadsorption with CytoSorb shows a decreased observed versus expected 28- day all-cause mortality in ICU patients with septic shock: a propensity-score-weighted retrospective study
}

\author{
Willem Pieter Brouwer ${ }^{1,2^{*}}$, Servet Duran ${ }^{3}$, Martijn Kuijper ${ }^{4}$ and Can Ince ${ }^{5}$
}

\begin{abstract}
Background and aims: Innovative treatment modalities have not yet shown a clinical benefit in patients with septic shock. To reduce severe cytokinaemia, CytoSorb as an add-on to continuous renal replacement therapy (CRRT) showed promising results in case reports. However, there are no clinical trials investigating outcomes.

Methods: In this investigator-initiated retrospective study, patients with septic shock were treated with CRRT + CytoSorb $(n=67)$ or CRRT alone $(n=49)$. The primary outcome was the 28-day all-cause mortality rate. Patients were weighted by stabilized inverse probability of treatment weights (SIPTW) to overcome differences in baseline characteristics.

Results: At the start of therapy, CytoSorb-treated patients had higher lactate levels $(p<0.001)$, lower mean arterial pressure $(p=0.007)$ and higher levels of noradrenaline $(p<0.001)$ compared to the CRRT group. For CytoSorb, the mean predicted mortality rate based on a SOFA of $13.8(n=67)$ was $75 \%(95 \% \mathrm{Cl} 71-79 \%)$, while the actual 28 -day mortality rate was $48 \%$ (mean difference $-27 \%, 95 \% \mathrm{Cl}-38$ to $-15 \%, p<0.001$ ). For CRRT, based on a SOFA of 12.8 $(n=49)$, the mean predicted versus observed mortality was $68 \%$ versus $51 \%$ (mean difference $-16.9 \%[95 \% \mathrm{Cl}-$ 32.6 to $-1.2 \%, p=0.035]$ ). By sIPTW analysis, patients treated with CytoSorb had a significantly lower 28-day mortality rate compared to CRRT alone (53\% vs. $72 \%$, respectively, $p=0.038$ ). Independent predictors of 28-day mortality in the CytoSorb group were the presence of pneumosepsis (adjusted odds ratio [aOR] 5.47, $p=0.029$ ), higher levels of lactate at the start of CytoSorb (aOR 1.15, $p=0.031$ ) and older age (aOR per 10 years 1.67, $p=0.034$ ).

Conclusions: CytoSorb was associated with a decreased observed versus expected 28-day all-cause mortality. By IPTW analysis, intervention with CytoSorb may be associated with a decreased all-cause mortality at 28 days compared to CRRT alone.
\end{abstract}

Keywords: Sepsis, Septic shock, Treatment, Hemofiltration, Cytosorb, Cytosorbent, Mortality, Outcome

\footnotetext{
* Correspondence: w.p.brouwer@erasmusmc.nl

${ }^{1}$ Department of Gastroenterology and Hepatology, Erasmus MC, University Medical Center Rotterdam, Dr Molewaterplein 15, Building NA-6, 3015, CE, Rotterdam, The Netherlands

${ }^{2}$ Department of Internal Medicine, Maasstad Ziekenhuis, Rotterdam, The

Netherlands

Full list of author information is available at the end of the article
}

(c) The Author(s). 2019 Open Access This article is distributed under the terms of the Creative Commons Attribution 4.0 International License (http://creativecommons.org/licenses/by/4.0/), which permits unrestricted use, distribution, and reproduction in any medium, provided you give appropriate credit to the original author(s) and the source, provide a link to the Creative Commons license, and indicate if changes were made. The Creative Commons Public Domain Dedication waiver (http://creativecommons.org/publicdomain/zero/1.0/) applies to the data made available in this article, unless otherwise stated. 


\section{Introduction}

Sepsis and septic shock are a major health burden worldwide leading to approximately 5 million deaths annually [1-3]. Although the reported incidence of sepsis varies widely and is notoriously unreliable, it is the leading cause of mortality globally, and its incidence currently is thought to rise due to aging populations, increasing comorbidity and greater recognition due to increasing awareness of this disease over the past decades $[4,5]$. Septic shock is thought to arise from a disrupted balance between pro-inflammatory and antiinflammatory cytokines in response to infection, ultimately leading to cell and organ dysfunction [5]. Recent advances in the field of sepsis treatment had variable success, presumably because sepsis is a very heterogeneous disease entity and therefore resists a one-size-fitsall approach. To date, only advancement in supportive care, such as timely delivery of antibiotics and early fluid resuscitation, has led to a significant improvement in the outcome of sepsis [4]. Other treatment modalities, such as continuous renal replacement therapy (CRRT) have not shown clinical benefit, although it was shown that cytokines such as tumour necrosis factor (TNF) $\alpha$ and interleukin (IL) $1 \beta$ could be cleared from serum [6-8].

Recently, CytoSorb has been developed and approved in Europe since 2011 for use in patients with severe cytokinaemia [9-11]. CytoSorb is a filter which can be used in addition to continuous renal replacement therapy (CRRT), and other devices such as hemodialysis, heart-lung machines and extracorporeal membrane oxygenation. It is a non-pyrogetic, sterile single-use filter for the removal of endotoxins and cytokines [10]. Since it is able to reduce circulating cytokines such as IL- $1 \beta$, TNF$\alpha$, IL- 6 and IL-10 by more than $90 \%$, CytoSorb is thought to have considerable impact on a derailed host response causing shock $[10,12]$. This treatment modality has shown promising results in animal studies $[13,14]$ and case reports [15-18]. Nevertheless, a recent randomized trial in patients with septic shock and acute lung injury (ALI) or acute respiratory distress syndrome (ARDS) assessed, but was not powered for mortality, and found no difference in clinical outcome [11, 19]. It is therefore unknown whether CytoSorb leads to a survival benefit.

Hence, the aim of the current study is to investigate whether the application of CytoSorb in addition to CRRT leads to a reduction in 28-day mortality compared to CRRT alone in patients with septic shock in the ICU, by using the inversed probability of treatment weights method.

\section{Patients and methods}

\section{Patients}

In this retrospective investigator-initiated study, patients admitted to the ICU of the Maasstad Hospital with septic shock [5] treated with CRRT with or without CytoSorb from Jan 01, 2014 - April 01, 2017, were initially eligible for inclusion. CytoSorb was initiated at the discretion of the treating intensive care physician. Indications for CytoSorb therapy were age 18-80 years and having a septic shock (see definitions below). Patients were treated per protocol, agreed upon by the staff of intensive care physicians. All patients in this study were treated with CRRT. Patients were excluded from the analysis in case the primary diagnosis was not septic shock (out of hospital cardiac arrest, rhabdomyolysis, intoxications, metabolic disturbances, kidney or heart failure with type 1 respiratory insufficiency requiring CRRT, or presence of active malignancy). Moreover, CytoSorb or CRRT was discontinued in case shock or renal function was improved. For the current study, to test the application of CytoSorb to CRRT in a clinical practice setting, there were no constraints to the timing of admission to the ICU, the severity of septic shock at the start of therapy and the eventual duration of therapy. Patients were treated per protocol as part of standard of care, i.e. no interventions were applied for the purpose of this study, and data was collected retrospectively. Patients who initiated on CytoSorb subsequent to CRRT were evaluated in the CytoSorb cohort. CytoSorb was used according to the manufacturer's protocol. It was placed in a blood-pump circuit with an optimal ultrafiltration rate of $250-400 \mathrm{~mL} / \mathrm{min}$. The CytoSorb filter was changed after $24 \mathrm{~h}$ of use.

\section{Definitions}

Septic shock was defined as a life-threatening organ dysfunction caused by a dysregulated host response to infection, identified by persisting hypotension requiring vasopressive medication to maintain mean arterial pressure $(\mathrm{MAP}) \geq 65 \mathrm{mmHg}$ and having a serum lactate level $>2 \mathrm{mmol} / \mathrm{L}$ despite adequate volume resuscitation [5]. Shock reversal was defined as a serum lactate level $\leq 2 \mathrm{mmol} / \mathrm{L}$ and discontinuation of vasopressive medication [20].

\section{Endpoints}

The primary endpoint was the 28-day all-cause mortality compared for CytoSorb versus CRRT alone. Secondary endpoints included the comparison between the observed 28-day mortality rate in the CytoSorb treatment group versus the predicted mortality according to the SOFA score [21, 22], and variables that predict mortality in the CytoSorb group. All-cause mortality was measured from ICU admission until 28 days after admission (irrespective of ICU, in-hospital or out of hospital mortality). 


\section{Statistical analysis}

For the first part, the predicted probability according to the SOFA score at the start of therapy $[21,22]$ was calculated for each individual and compared with the observed mortality rate in the CytoSorb group using a paired $T$-test.

For the second part, all evaluations were carried out using the inverse probability of treatment weights (IPTW), including a stabilizing method to avoid bias from extreme weights [23, 24]. Stabilized IPTW (sIPTW) is applied to overcome differences in baseline patient characteristics, to mimic a randomized controlled trial. Weights are based on the propensity score to create a synthetic sample in which the baseline variables are independent of the treatment assignment. First, all variables in the dataset were tested for their association with either CytoSorb or CRRT treatment. From this analysis, factors associated with the treatment in univariate analysis were selected to construct a multivariable model to estimate the probability of being treated with CRRT or CytoSorb. Second, the probability of being treated with CRRT or CytoSorb was estimated using the following baseline factors in a logistic regression analysis: age, SOFA score at the start of therapy, lactate level at the start of therapy, dosage of vasopressive medication (in $\mu \mathrm{g} / \mathrm{kg} / \mathrm{min}$ ) at the start of therapy, known comorbidity, surgery just prior to or during ICU admission and origin of sepsis. These factors are also associated with the primary outcome $[5,21$, 25]. Third, patients were weighted by the inverse of this propensity, which was stabilized prior to the analyses using the estimated marginal means of the calculated propensity [23]. The absolute standardized difference for variables between the two treatment groups were calculated using Cohen's D and graphically inspected (Additional file 1: Figure S1). The association between CytoSorb or CRRT with clinical outcome at 28 days was then estimated by chisquare analysis. Fourth, to account for variables still showing imbalance after adjustment by sIPTW, multivariable logistic regression was applied using both the stabilized weights and adjusting for unbalanced variables [24]. Factors associated with 28-day mortality in CytoSorb-treated patients were analysed as well. For this, factors with a $p$ value $<0.1$ in univariate analysis were considered for multivariable regression analyses. First, a full multivariable model was constructed where all possible important variables were forced into the model. Second, using the same variables from the full model, the final model was constructed with the backstep likelihood ratio method. SPSS version 22.0 (SPSS Inc., Chicago, IL, USA) was used to perform statistical analyses. All statistical tests were two-sided and evaluated at the 0.05 level of significance.

\section{Results}

\section{Patient characteristics}

In total, 210 patients were treated, of which 101 with CytoSorb and 109 with CRRT only. Of the patients treated with CytoSorb, 67 were treated because of septic shock, versus 49 for CRRT. Figure 1 shows the patient selection and reasons for exclusion. The patient characteristics can be found in Table 1. It was observed that patients treated with CytoSorb had worse hemodynamic characteristics when compared to CRRT alone. CytoSorb-treated patients had higher lactate levels both at admission $(p=0.027)$ and at the start of therapy $(p<$ 0.001 ), were administered higher levels of noradrenaline $(p<0.001)$ and had lower mean arterial pressure $(p=$ 0.007). Patients were treated with CRRT for a mean duration of 4.96 (SE 0.63) days in the CRRT group and 4.97 (SE 0.55) days in the CytoSorb group $(p=0.990)$. In the CytoSorb group, patients were treated with CytoSorb added to CRRT for a mean duration of 2.34 (SE 0.16) days and with CRRT only for a mean 2.66 (SE 0.52) days. The mean duration from ICU admission to the start of treatment was 2.1 (SE 0.36) versus 1.66 (SE 0.38) days $(p=0.416)$ for CRRT versus CytoSorb, respectively. In total, 88\% $(n=59)$ patients commenced CytoSorb directly together with CRRT because of septic shock (i.e. no delay between CRRT and CytoSorb), 4 patients had a delay in the start of CytoSorb after CRRT of 1 day and another 4 had a delay of $>1$ day. Moreover, the mean duration from hospital admission to the start of treatment did not differ between the groups (4.7 versus 3.8 days, $p=0.306$ ). After adjustment with sIPTW, the CRRT and CytoSorb groups were largely comparable (Table 1 and Additional file 1: Figure S1).

\section{CytoSorb treatment: observed versus predicted 28-day mortality}

When no correction for baseline variables was applied, it was shown that the 28-day all-cause mortality rate was similar for CytoSorb versus CRRT (47.8\% versus $51.0 \%$, $p=0.729$, Fig. 2). For the CytoSorb group, the mean SOFA score at the start of therapy was 13.8 (SE 2.8), and the delta SOFA score (admission to treatment) was 2.1 (SE 0.41). On the basis of the SOFA score, the mean predicted mortality rate was $74.5 \%$ (95\%CI $70.7-79.0 \%)$ [21], while the actual 28-day mortality rate was $47.8 \%$ (95\%CI 35.7-59.8\%), corresponding to a mean difference of $-26.8 \%(95 \% \mathrm{CI}-38.2$ to $-15.3 \%, p<0.001)$. For the CRRT only group, the mean SOFA score was 12.8 (SE 3.2 ), corresponding to a mean predicted mortality rate of $67.9 \%$ (95\% CI $60.7-75.2 \%$ ), while the observed mortality rate was $51.0 \%(95 \% \mathrm{CI} 36.9-65.2 \%)$ with a mean difference $-16.9 \%(95 \% \mathrm{CI}-32.6$ to $-1.2 \%, p=0.035)$.

\section{CytoSorb is associated with a reduced 28-day mortality: sIPTW analysis}

By sIPTW chi-square analysis, the 28-day mortality significantly differed for CytoSorb versus CRRT: $53.0 \%$ versus $72.3 \%$, respectively $(p=0.038$, Fig. 2$)$. By sIPTW 


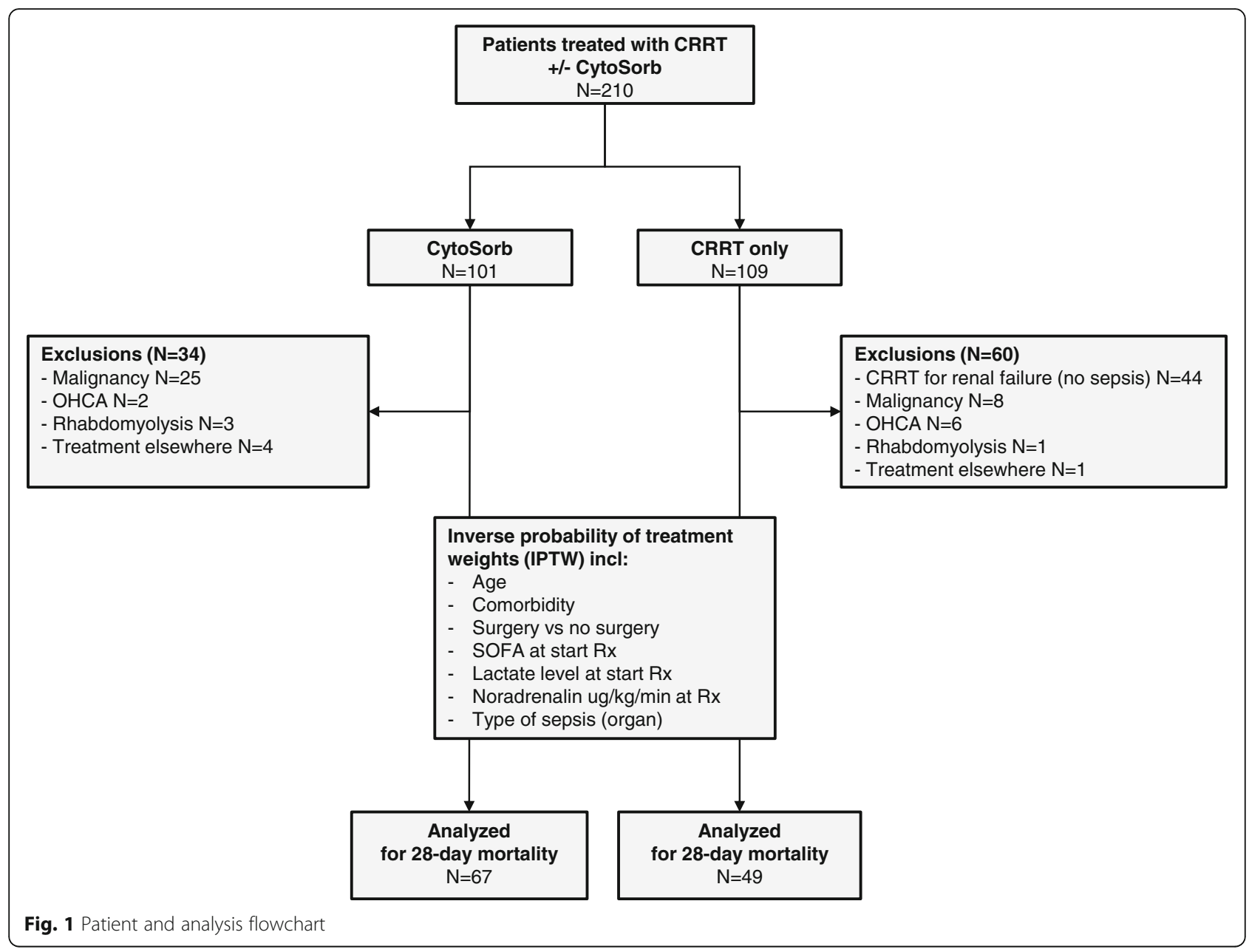

multivariable analysis additionally adjusted for MAP and chronic kidney disease (CKD), CytoSorb treatment was also significantly associated with an improved 28day outcome (OR 0.37, 95\% confidence interval [CI] $0.15-0.92, p=0.032)$. In this analysis, CKD was not associated with 28 -day mortality (OR 1.93 , 95\%CI $0.69-$ $5.44, p=0.214$ ), and a higher MAP was associated with a lower chance of mortality (OR $0.95,95 \%$ CI $0.92-0.98$, $p=0.003)$.

\section{Factors associated with 28-day mortality for CytoSorb therapy}

Factors at the start of therapy significantly associated with mortality at 28 days for CytoSorb-treated patients were the SOFA score (OR 1.28, 95\%CI 1.05-1.56, $p=$ 0.014), lactate levels (OR 1.16, 95\%CI 1.03-1.30, $p=$ 0.014 ), noradrenaline levels (OR 2.60, 95\%CI 1.15-5.87, $p=0.021$ ), and older age (OR 1.67 per 10 years older, $p=0.002$, Table 2). For patients with mortality versus those who survived, initiation of CytoSorb was 1.13 (SE 0.35 ) versus 2.14 (SE 0.65$)$ days, respectively $(p=0.18)$. To investigate whether CytoSorb treatment may have been initiated earlier in patients with worse septic shock, interactions between therapy timing and lactate levels or SOFA score at the start of therapy were applied, which were found non-significant $(p=0.538$ and $p=0.930$, respectively). By multivariable analysis, independent predictors of 28-day mortality in the CytoSorb group were older age (OR per 10 years older 1.67, 95\%CI 1.00-2.70, $p=0.034)$, higher levels of lactate at the start of therapy (OR 1.15, 95\%CI 1.01-1.30, $p=0.031$ ) and pneumosepsis (OR 5.47, 95\%CI 1.19-25.19, $p=0.029$ ). Other factors were not independently associated with mortality (Table 2).

\section{Discussion}

In this investigator-initiated retrospective study, we have shown for the first time that CytoSorb therapy may improve the 28-day mortality for patients with septic shock, compared to CRRT. To our knowledge, this represents the largest cohort of septic shock patients treated with CytoSorb therapy in which mortality was assessed as a primary outcome. The observed mortality rate for CytoSorb therapy was significantly below the predicted 
Table 1 Patient characteristics (at ICU admission and at the start of CytoSorb or CRRT)

\begin{tabular}{|c|c|c|c|c|}
\hline Characteristics & CytoSorb $(N=67)$ & CRRT only $(n=48)$ & Unadjusted $p$ value & sIPTW-adjusted $p$ value \\
\hline \multicolumn{5}{|l|}{ Demography } \\
\hline Age, years (Mean, SD) & $61.1(14.7)$ & $68.7(9.6)$ & 0.001 & 0.126 \\
\hline Male, $n(\%)$ & $37(55 \%)$ & $30(61 \%)$ & 0.522 & 0.021 \\
\hline \multicolumn{5}{|l|}{ Comorbidity, n (\%) } \\
\hline Any comorbidity & $43(64 \%)$ & $42(88 \%)$ & 0.002 & 0.601 \\
\hline Diabetes mellitus type 2 & $14(21 \%)$ & $20(41 \%)$ & 0.024 & 0.778 \\
\hline Hypertension & $23(34 \%)$ & $26(53 \%)$ & 0.046 & 0.409 \\
\hline Coronary heart disease & $9(13 \%)$ & $8(16 \%)$ & 0.667 & 0.064 \\
\hline Heart failure (systolic/diastolic) & $4(6 \%)$ & $10(20 \%)$ & 0.030 & 0.501 \\
\hline Prior chronic kidney disease & $8(12 \%)$ & $18(37 \%)$ & 0.003 & 0.002 \\
\hline Peripheral artery disease & $10(15 \%)$ & $8(16 \%)$ & 0.839 & 0.021 \\
\hline Cerebrovascular accident & $6(9 \%)$ & $3(6 \%)$ & 0.577 & 0.369 \\
\hline COPD & $8(12 \%)$ & $10(20 \%)$ & 0.233 & 0.002 \\
\hline \multicolumn{5}{|l|}{ Primary diagnosis, n (\%) } \\
\hline Abdominal sepsis & $31(46 \%)$ & $12(25 \%)$ & 0.014 & 0.870 \\
\hline Pneumosepsis & $14(21 \%)$ & $21(43 \%)$ & 0.014 & 0.275 \\
\hline Urosepsis & $2(3 \%)$ & $6(12 \%)$ & 0.078 & 0.217 \\
\hline Cutaneous/arthritis & $9(13 \%)$ & $3(6 \%)$ & 0.182 & 0.064 \\
\hline Vascular sepsis & $5(8 \%)$ & 0 & 0.024 & 0.026 \\
\hline Cerebral sepsis & 0 & $1(2 \%)$ & 0.322 & 0.475 \\
\hline Sepsis (unknown cause) & $6(9 \%)$ & $6(12 \%)$ & 0.569 & 0.755 \\
\hline \multicolumn{5}{|l|}{ Admission } \\
\hline Surgical (otherwise medical) & $27(40 \%)$ & $6(12 \%)$ & $<0.001$ & 0.994 \\
\hline Days on ICU & $9(2-19)$ & $9(3-13)$ & 0.783 & 0.463 \\
\hline \multicolumn{5}{|l|}{ Hemodynamics (Mean, SD) } \\
\hline Lactate at admission & $6.4(5.1)$ & $4.4(4.4)$ & 0.027 & 0.421 \\
\hline Lactate at the start of therapy & $6.9(5.6)$ & $2.9(3.1)$ & $<0.001$ & 0.544 \\
\hline Noradrenaline $(\mu \mathrm{g} / \mathrm{kg} / \mathrm{min}) \mathrm{adm}$. & $0.48(0.55)$ & $0.29(0.40)$ & 0.052 & 0.073 \\
\hline Noradrenaline $(\mu \mathrm{g} / \mathrm{kg} / \mathrm{min}) \mathrm{Rx}$ & $0.96(0.73)$ & $0.28(0.36)$ & $<0.001$ & 0.769 \\
\hline Total duration noradrenaline (days) & $3(1-5)$ & $3(1-5)$ & 0.979 & 0.222 \\
\hline Duration noradrenaline from Rx (days) & $2(1-3)$ & $1(0-2)$ & 0.694 & 0.989 \\
\hline MAP at admission & $73(19)$ & $74(20)$ & 0.748 & 0.418 \\
\hline MAP at the start of therapy & $69(15)$ & $77(18)$ & 0.007 & 0.019 \\
\hline \multicolumn{5}{|l|}{ Prognostic scores } \\
\hline SOFA ICU admission & $11.7(3.3)$ & $11.8(3.5)$ & 0.907 & 0.854 \\
\hline SOFA at the start of treatment & $13.8(2.8)$ & $12.8(3.2)$ & 0.067 & 0.239 \\
\hline
\end{tabular}

risk of death according to the SOFA score [21, 22]. For CytoSorb, factors associated with a higher chance of 28day mortality were older age, higher lactate levels at the start of therapy and pneumosepsis.

In the current study, it was investigated whether CytoSorb improves survival when compared to CRRT alone in an IPTW analysis. Per protocol, patients were only treated with CytoSorb in case of septic shock, at the discretion of the treating intensive care physician. As a result, it was found that at the start of therapy, patients treated with CytoSorb had a worse septic shock than those treated with CRRT alone as shown by higher lactate and noradrenaline levels, and lower mean arterial blood pressure. Despite that CytoSorb-treated patients had a worse shock, in an unbalanced analysis, the mortality rate was comparable to patients treated with CRRT alone. However, it could be argued that patients treated with CRRT alone were older with more comorbidities 

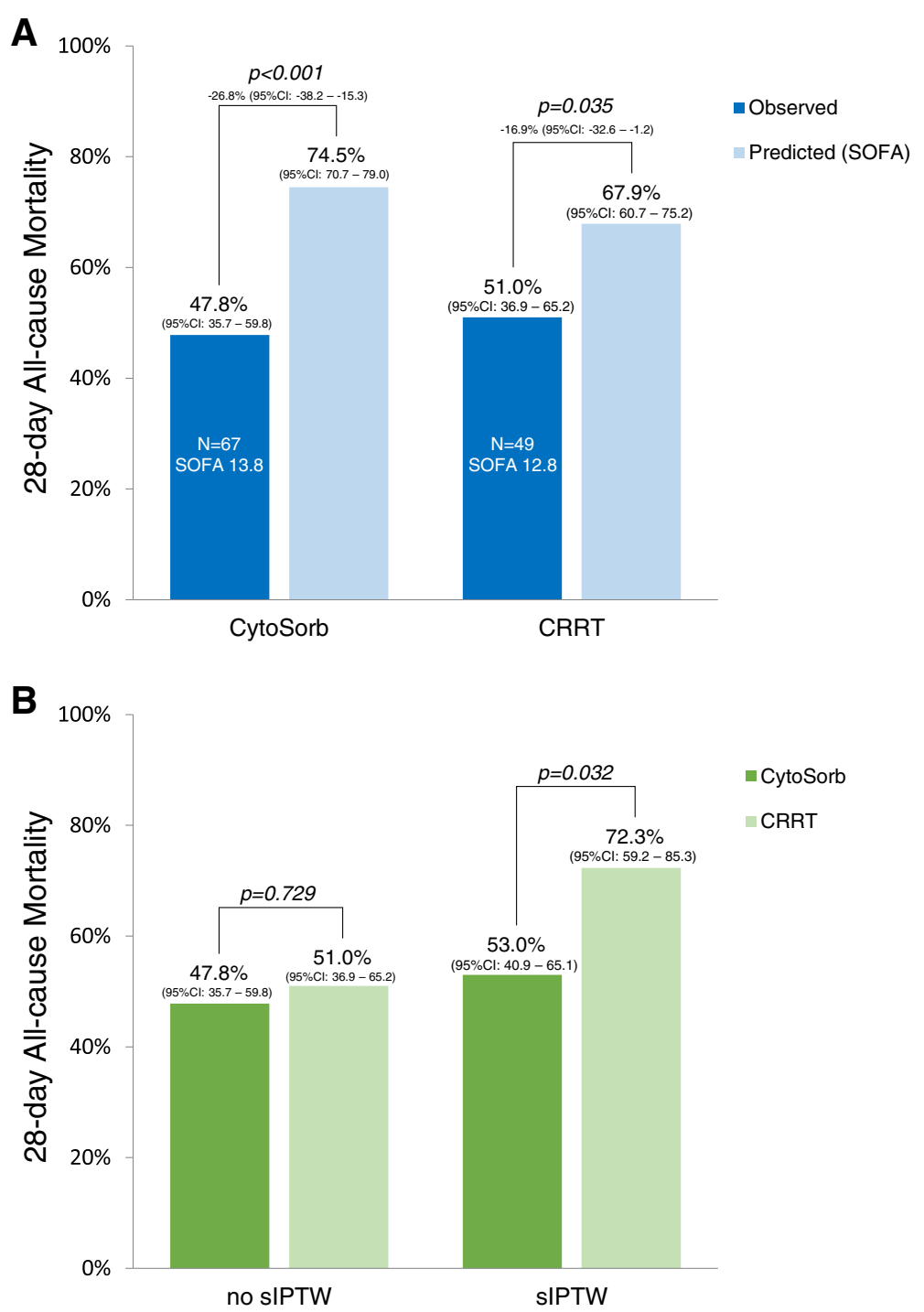

Fig. 2 a Observed versus predicted mortality rate according to the SOFA score for CytoSorb- and CRRT-treated patients. b CytoSorb is associated with a reduced 28-day mortality in SIPTW analysis

and more often having non-surgical sepsis. Therefore, observed versus predicted mortality rates were analysed within treatment groups. This analysis showed that the mortality rates in this study were lower than that predicted by the SOFA score at the start of therapy [21]. The results of our study are in line with a previous prospective study in 20 patients with septic shock treated with CytoSorb, where a 28 -day mortality rate of $45 \%$ was reported as well [20].

It is important to investigate factors which are associated with survival for CytoSorb treatment. For the current cohort, next to older age, we found that higher lactate levels at baseline of CytoSorb therapy were associated with a worse outcome. Indeed, these factors are components of the SOFA score itself. Apparently, particularly the measures of the hemodynamic components of the SOFA score showed the strongest association with outcome. Hence, it seems reasonable to assume that CytoSorb therapy should be initiated as early as possible in the disease course. Nonetheless, 8 (12\%) patients received CytoSorb at least 1 day after CRRT was already started. We performed a sensitivity analysis in which we excluded these patients and found no deviations from the main results.

In our cohort, it was found that CytoSorb therapy did not seem to provide with a survival benefit for patients with pneumosepsis. This may in part be due to combined difficulties in adequate ventilation and/or presence of ALI or ARDS in these severely ill patients. These findings are underlined by a recent randomized trial which assessed IL-6 levels as a primary outcome in patients with ALI and ARDS and found no survival benefit in 
Table 2 Regression analysis for mortality at 28 days for CytoSorb-treated patients

\begin{tabular}{|c|c|c|c|c|c|c|}
\hline \multirow[t]{2}{*}{ Variables } & \multicolumn{2}{|l|}{ Univariable } & \multicolumn{2}{|l|}{ Full multivariable model (forced) } & \multicolumn{2}{|l|}{ Final multivariable model (bstep LR) } \\
\hline & OR $(95 \% \mathrm{Cl})$ & $p$ & OR $(95 \% \mathrm{Cl})$ & $p$ & OR $(95 \% \mathrm{Cl})$ & $p$ \\
\hline Age per 10 years older & $1.99(1.3-3.1)$ & 0.002 & $1.64(1.0-2.7)$ & 0.050 & $1.67(1.0-2.7)$ & 0.034 \\
\hline Female gender & $1.50(0.6-4.0)$ & 0.412 & - & & - & \\
\hline Body mass index & $0.96(0.9-1.0)$ & 0.260 & - & & - & \\
\hline SOFA admission & $1.20(1.0-1.4)$ & 0.041 & - & & - & \\
\hline SOFA at the start of treatment & $1.28(1.1-1.6)$ & 0.014 & $1.10(0.8-1.4)$ & 0.505 & - & \\
\hline MAP admission & $0.99(0.9-1.0)$ & 0.460 & - & & - & \\
\hline MAP at the start of treatment & $0.96(0.9-1.0)$ & 0.051 & $0.99(1.0-1.03)$ & 0.739 & - & \\
\hline Lactate admission & $1.07(0.9-1.2)$ & 0.180 & - & & - & \\
\hline Lactate at the start of treatment & $1.16(1.0-1.3)$ & 0.014 & $1.13(1.0-1.3)$ & 0.108 & $1.15(1.0-1.3)$ & 0.031 \\
\hline Noradrenaline admission & $2.25(0.8-6.0)$ & 0.106 & - & & - & \\
\hline Noradrenaline treatment & $2.60(1.2-5.9)$ & 0.021 & $0.98(0.3-2.8)$ & 0.973 & - & \\
\hline Noradrenaline duration & $1.02(0.9-1.2)$ & 0.731 & - & & - & \\
\hline Admission ICU to treatment & $0.88(0.7-1.1)$ & 0.216 & - & & - & \\
\hline Admission hospital to treatment & $0.98(0.9-1.1)$ & 0.675 & - & & - & \\
\hline Surgery & $0.37(0.1-1.0)$ & 0.055 & $0.43(0.1-1.6)$ & 0.206 & - & \\
\hline Type of sepsis: & - & & - & & - & \\
\hline Pneumosepsis & $3.52(1.0-12.7)$ & 0.054 & $2.83(0.5-16.9)$ & 0.254 & $5.47(1.2-25.2)$ & 0.029 \\
\hline Abdominal sepsis & $0.51(0.2-1.3)$ & 0.171 & - & & - & \\
\hline Cutaneous sepsis & $0.27(0.1-1.4)$ & 0.117 & - & & - & \\
\hline Vascular sepsis & $4.86(0.5-46.0)$ & 0.168 & - & & - & \\
\hline Sepsis unknown cause & $2.36(0.4-13.8)$ & 0.343 & - & & - & \\
\hline History of: & - & & - & & - & \\
\hline Diabetes mellitus & $1.61(0.5-5.3)$ & 0.432 & - & & - & \\
\hline Hypertension & $1.31(0.5-3.6)$ & 0.601 & - & & - & \\
\hline COPD & $1.97(0.4-9.0)$ & 0.380 & & & & \\
\hline Coronary artery disease & $0.86(0.2-3.5)$ & 0.831 & - & & - & \\
\hline Heart failure & NA & 0.999 & & & & \\
\hline Chronic kidney disease & $1.98(0.4-9.0)$ & 0.380 & - & & - & \\
\hline Hemodialysis/peritoneal & $1.10(0.1-18.3)$ & 0.949 & - & & - & \\
\hline CVA & $1.10(0.2-5.9)$ & 0.908 & - & & - & \\
\hline Peripheral artery disease & $2.99(0.7-12.7)$ & 0.139 & - & & - & \\
\hline
\end{tabular}

MAP mean arterial pressure, ICU intensive care unit, COPD chronic obstructive pulmonary disease, CVA cerebrovascular accident Statistically significant data are italicized

these patients as a secondary (but not powered for) outcome measure [11].

Since the current study is a retrospective data analysis, there is inherent bias to take into account. Importantly, by applying IPTW, confounding by indication was as much as possible eradicated [24]. Still, chance of residual confounding remains, and some variables were imbalanced at the start of therapy. Only MAP was associated with the primary endpoint, which was accounted for by multivariable analysis. Moreover, the precise amount of fluid balances and ultrafiltration rates were not available for both treatment groups, which was complicated because this would need a dynamic statistical analysis. Indeed, fluid balances may be important measures as several studies show an association with more positive fluid balances and mortality [26, 27], but there are also controversial findings with studies showing an association between higher fluid balance and improved survival $[28,29]$, and even no differences in early goaldirected therapy [30-32]. It should be underlined that it is uncertain whether the association between a positive fluid balance and mortality is a true dose-response causal relationship. Even though the omission of data on the fluid balance may be a limitation of the study, both 
groups received exactly the same standard of care fluid resuscitation protocols since this is a mono-centre study. On this basis, both groups are not expected to differ accordingly.

Albeit patients had a severe refractory septic shock and were treated with CytoSorb as a last -resort, one may argue that this treatment modality could have potential detrimental effects. One of these effects may be that CytoSorb could filter out antibiotics leaving patients exposed to levels below the therapeutic range [33]. In the current study, we did not have antibiotic levels available. Nonetheless, there were no observations or indications of excessive need for antibiotics or persistence of infections in the CytoSorb group. Moreover, septic shock originates from a severe host-response derailment and endotoxinaemia and as such these patients may benefit more from CytoSorb therapy than antibiotics alone, which is underlined by the current data. Our data also shows that CytoSorb leads to a better outcome in patients with less severe lactataemia. Given possible antibiotic filtration, caution is warranted since the positive effect may be tipped towards a more negative effect if CytoSorb therapy is initiated too early. Lastly, a recent pilot study showed positive effects on lactate and procalcitonin when CytoSorb was used as a stand-alone therapy [34]. We did not have procalcitonin or interleukin levels available in our study. Future randomized trials comparing CytoSorb to CRRT should further elucidate the effect on interleukin, procalcitonin and antibiotic levels, and what the timing and duration of CytoSorb therapy should be.

\section{Conclusion}

We have shown, to our knowledge, in the largest cohort of septic shock patients to date, that CytoSorb treatment may lead to an improved 28-day survival compared to CRRT alone, both on basis of observed versus predicted mortality rates as well as by IPTW. The current data should be further corroborated by randomized clinical trials.

\section{Additional file}

Additional file 1: Figure S1. Absolute standardized differences between CRRT and CytoSorb for variables before and after weighting. (PDF $403 \mathrm{~kb})$

\section{Abbreviations}

ALI: Acute lung injury; ARDS: Acute respiratory distress syndrome; CKD: Chronic kidney disease; CRRT: Continuous renal replacement therapy; ECMO: Extracorporeal membrane oxygenation; IL: Interleukin; MAP: Mean arterial pressure; sIPTW: Stabilized inverse probability of treatment weights; ICU: Intensive care unit; OR: Odds ratio; LR: Likelihood ratio; SD: Standard deviation; SE: Standard error; TNF: Tumour necrosis factor

\section{Acknowledgements}

Not applicable.

\section{Authors' contributions}

WPB contributed to the study design, data collection, statistical methods and analysis, writing of manuscript, and approval of the final version. SD and $\mathrm{Cl}$ contributed to the study design, critical review of the manuscript, and approval of the final version. MK contributed to the critical review of the manuscript, statistical methodology, and approval of the final version. WPB, SD and $\mathrm{Cl}$ had full access to all of the data in the study and take responsibility for the integrity of the data and the accuracy of the data analysis.

\section{Funding}

None.

\section{Availability of data and materials}

The dataset used and analysed during the current study are available from the corresponding author on reasonable request.

\section{Ethics approval and consent to participate}

All patients, their relatives or legal representatives gave their informed consent for the admission to the ICU and to undergo specific interventions. Patients were not re-contacted for the purpose of this study. To re-contact patients for informed consent for the current retrospective chart study would introduce a serious bias because a large proportion is out of follow-up or deceased. All procedures were carried out per standard of care per protocol. This retrospective cohort study was performed in accordance with the protocol, the principles of the Declaration of Helsinki 1964 as modified by the 64th WMA General Assembly, Fortaleza, Brazil, October 2013, with notes of clarification on paragraph 29 and 30 added by the WMA General Assembly, and the local national laws governing the conduct of clinical research studies. The study was approved by the ethics committee of the Maasstad Hospital Rotterdam, the Netherlands (MZ0582-07-14, L2017-030).

\section{Consent for publication}

Not applicable.

\section{Competing interests}

WPB, MK and SD declare that they have no competing interests. Cl has received a grant from CytoSorb to commence a randomized controlled trial on the effect of the adsorber on the microcirculation of critically ill patients at the department of Intensive Care of the Erasmus Medical Center Rotterdam.

\section{Author details}

'Department of Gastroenterology and Hepatology, Erasmus MC, University Medical Center Rotterdam, Dr Molewaterplein 15, Building NA-6, 3015, CE, Rotterdam, The Netherlands. ${ }^{2}$ Department of Internal Medicine, Maasstad Ziekenhuis, Rotterdam, The Netherlands. ${ }^{3}$ Department of Intensive Care Medicine, Maasstad Ziekenhuis, Rotterdam, The Netherlands. ${ }^{4}$ Science board, Maasstad Ziekenhuis, Rotterdam, The Netherlands. ${ }^{5}$ Department of Intensive Care Medicine, Erasmus MC, University Medical Center Rotterdam, Rotterdam, The Netherlands.

Received: 16 June 2019 Accepted: 28 August 2019

Published online: 18 September 2019

References

1. Lozano R, Naghavi M, Foreman K, Lim S, Shibuya K, Aboyans V, Abraham J, Adair T, Aggarwal R, Ahn SY, et al. Global and regional mortality from 235 causes of death for 20 age groups in 1990 and 2010: a systematic analysis for the Global Burden of Disease Study 2010. Lancet. 2012;380(9859):2095-128.

2. Vincent JL, Marshall JC, Namendys-Silva SA, Francois B, Martin-Loeches I, Lipman J, Reinhart K, Antonelli M, Pickkers P, Njimi H, et al. Assessment of the worldwide burden of critical illness: the intensive care over nations (ICON) audit. Lancet Respir Med. 2014;2(5):380-6.

3. Fleischmann C, Scherag A, Adhikari NK, Hartog CS, Tsaganos T, Schlattmann P, Angus DC, Reinhart K. Assessment of global incidence and mortality of hospital-treated sepsis. Current estimates and limitations. Am J Respir Crit Care Med. 2016;193(3):259-72. 
4. Levy MM, Artigas A, Phillips GS, Rhodes A, Beale R, Osborn T, Vincent JL, Townsend S, Lemeshow S, Dellinger RP. Outcomes of the Surviving Sepsis Campaign in intensive care units in the USA and Europe: a prospective cohort study. Lancet Infect Dis. 2012;12(12):919-24.

5. Singer M, Deutschman CS, Seymour CW, Shankar-Hari M, Annane D, Bauer M, Bellomo R, Bernard GR, Chiche JD, Coopersmith CM, et al. The Third International Consensus Definitions for Sepsis and Septic Shock (Sepsis-3). JAMA. 2016:315(8):801-10.

6. Bellomo R, Tipping P, Boyce N. Continuous veno-venous hemofiltration with dialysis removes cytokines from the circulation of septic patients. Crit Care Med. 1993;21(4):522-6.

7. Rimmele T, Kellum JA. Clinical review: blood purification for sepsis. Crit Care. 2011;15(1):205

8. Hoffmann JN, Faist E. Removal of mediators by continuous hemofiltration in septic patients. World J Surg. 2001;25(5):651-9.

9. Houschyar KS, Pyles MN, Rein S, Nietzschmann I, Duscher D, Maan ZN, Weissenberg K, Philipps HM, Strauss C, Reichelt B, et al. Continuous hemoadsorption with a cytokine adsorber during sepsis - a review of the literature. Int J Artif Organs. 2017;40(5):205-11.

10. Gruda MC, Ruggeberg KG, O'Sullivan P, Guliashvili T, Scheirer AR, Golobish TD, Capponi VJ, Chan PP. Broad adsorption of sepsis-related PAMP and DAMP molecules, mycotoxins, and cytokines from whole blood using CytoSorb(R) sorbent porous polymer beads. PLoS One. 2018;13(1):e0191676.

11. Schadler D, Pausch C, Heise D, Meier-Hellmann A, Brederlau J, Weiler N, Marx G, Putensen C, Spies C, Jorres A, et al. The effect of a novel extracorporeal cytokine hemoadsorption device on IL-6 elimination in septic patients: a randomized controlled trial. PLoS One. 2017;12(10):e0187015.

12. Malard B, Lambert C, Kellum JA. In vitro comparison of the adsorption of inflammatory mediators by blood purification devices. Intensive Care Med Exp. 2018;6(1):12.

13. Peng $Z Y$, Carter MJ, Kellum JA. Effects of hemoadsorption on cytokine removal and short-term survival in septic rats. Crit Care Med. 2008;36(5): 1573-7

14. Linden K, Scaravilli V, Kreyer SF, Belenkiy SM, Stewart IJ, Chung KK, Cancio LC, Batchinsky Al. Evaluation of the Cytosorb hemoadsorptive column in a pig model of severe smoke and burn injury. Shock (Augusta, Ga). 2015;44(5): 487-95.

15. Basu R, Pathak S, Goyal J, Chaudhry R, Goel RB, Barwal A. Use of a nove hemoadsorption device for cytokine removal as adjuvant therapy in a patient with septic shock with multi-organ dysfunction: a case study. Indian J Critical Care Med. 2014;18(12):822-4.

16. Hetz H, Berger R, Recknagel P, Steltzer H. Septic shock secondary to betahemolytic streptococcus-induced necrotizing fasciitis treated with a novel cytokine adsorption therapy. Int J Artif Organs. 2014;37(5):422-6.

17. Bruenger F, Kizner L, Weile J, Morshuis M, Gummert JF. First successful combination of ECMO with cytokine removal therapy in cardiogenic septic shock: a case report. Int J Artif Organs. 2015:38(2):113-6.

18. Hinz B, Jauch O, Noky T, Friesecke S, Abel P, Kaiser R. CytoSorb, a novel therapeutic approach for patients with septic shock: a case report. Int J Artif Organs. 2015;38(8):461-4

19. Friesecke S, Trager K, Schittek GA, Molnar Z, Bach F, Kogelmann K, Bogdanski R, Weyland A, Nierhaus A, Nestler F, et al. International registry on the use of the CytoSorb(R) adsorber in ICU patients: study protocol and preliminary results. Med Klin Intensivmed Notfmed. 2017. https://doi.org/10. 1007/s00063-017-0342-5.

20. Friesecke S, Stecher SS, Gross S, Felix SB, Nierhaus A. Extracorporeal cytokine elimination as rescue therapy in refractory septic shock: a prospective single-center study. J Artif Organs. 2017;20(3):252-9.

21. Ferreira FL, Bota DP, Bross A, Melot C, Vincent JL. Serial evaluation of the SOFA score to predict outcome in critically ill patients. JAMA. 2001;286(14): 1754-8

22. de Grooth HJ, Geenen IL, Girbes AR, Vincent JL, Parienti JJ, Oudemans-van Straaten HM. SOFA and mortality endpoints in randomized controlled trials: a systematic review and meta-regression analysis. Crit Care. 2017;21(1):38.

23. Harder VS, Stuart EA, Anthony JC. Propensity score techniques and the assessment of measured covariate balance to test causal associations in psychological research. Psychol Methods. 2010;15(3):234-49.

24. Robins JM, Hernan MA, Brumback B. Marginal structural models and causal inference in epidemiology. Epidemiology. 2000;11(5):550-60.

25. Vincent JL, Moreno R, Takala J, Willatts S, De Mendonca A, Bruining H, Reinhart CK, Suter PM, Thijs LG. The SOFA (Sepsis-related Organ Failure
Assessment) score to describe organ dysfunction/failure. On behalf of the Working Group on Sepsis-Related Problems of the European Society of Intensive Care Medicine. Intensive Care Med. 1996;22(7):707-10.

26. Vincent JL, Sakr Y, Sprung CL, Ranieri VM, Reinhart K, Gerlach H, Moreno R, Carlet J, Le Gall JR, Payen D, et al. Sepsis in European intensive care units: results of the SOAP study. Crit Care Med. 2006:34(2):344-53.

27. Silva JM Jr, de Oliveira AM, Nogueira FA, Vianna PM, Pereira Filho MC, Dias LF, Maia VP, Neucamp Cde S, Amendola CP, Carmona MJ, et al. The effect of excess fluid balance on the mortality rate of surgical patients: a multicenter prospective study. Crit Care. 2013;17(6):R288.

28. Smith SH, Perner A. Higher vs. lower fluid volume for septic shock: clinical characteristics and outcome in unselected patients in a prospective, multicenter cohort. Crit Care. 2012;16(3):R76.

29. Lee SJ, Ramar K, Park JG, Gajic O, Li G, Kashyap R. Increased fluid administration in the first three hours of sepsis resuscitation is associated with reduced mortality: a retrospective cohort study. Chest. 2014;146(4): 908-15.

30. Pro Cl, Yealy DM, Kellum JA, Huang DT, Barnato AE, Weissfeld LA, Pike F, Terndrup T, Wang HE, Hou PC, et al. A randomized trial of protocol-based care for early septic shock. N Engl J Med. 2014;370(18):1683-93.

31. Investigators A, Group ACT, Peake SL, Delaney A, Bailey M, Bellomo R, Cameron PA, Cooper DJ, Higgins AM, Holdgate A, et al. Goal-directed resuscitation for patients with early septic shock. N Engl J Med. 2014; 371(16):1496-506.

32. Mouncey PR, Osborn TM, Power GS, Harrison DA, Sadique MZ, Grieve RD, Jahan R, Harvey SE, Bell D, Bion JF, et al. Trial of early, goal-directed resuscitation for septic shock. N Engl J Med. 2015;372(14):1301-11.

33. Burger R, Guidi M, Calpini V, Lamoth F, Decosterd L, Robatel C, Buclin T, Csajka C, Marchetti O. Effect of renal clearance and continuous renal replacement therapy on appropriateness of recommended meropenem dosing regimens in critically ill patients with susceptible life-threatening infections. J Antimicrob Chemother. 2018;73(12):3413-22. https://doi.org/10. 1093/jac/dky370.

34. Hawchar F, Laszlo I, Oveges N, Trasy D, Ondrik Z, Molnar Z. Extracorporeal cytokine adsorption in septic shock: a proof of concept randomized, controlled pilot study. J Crit Care. 2019;49:172-8.

\section{Publisher's Note}

Springer Nature remains neutral with regard to jurisdictional claims in published maps and institutional affiliations.

\section{Ready to submit your research? Choose BMC and benefit from:}

- fast, convenient online submission

- thorough peer review by experienced researchers in your field

- rapid publication on acceptance

- support for research data, including large and complex data types

- gold Open Access which fosters wider collaboration and increased citations

- maximum visibility for your research: over $100 \mathrm{M}$ website views per year

At $\mathrm{BMC}$, research is always in progress.

Learn more biomedcentral.com/submissions 